CRYSTALLOGRAPHIC COMMUNICATIONS

ISSN 2056-9890

Received 9 December 2019

Accepted 17 December 2019

Edited by C. Rizzoli, Universita degli Studi di Parma, Italy

Keywords: crystal structure; oxybis Schiff base; intermolecular interaction.

CCDC reference: 1445336

Supporting information: this article has supporting information at journals.iucr.org/e

\section{Bis\{4-[(2-hydroxy-5-methoxy-3-nitrobenzylidene)- amino]phenyl\} ether}

\author{
Md. Azharul Arafath, ${ }^{\mathrm{a} *}$ Huey Chong Kwong, ${ }^{\mathrm{b}}$ Farook Adam, ${ }^{\mathrm{c}}$ Md. Mohiuddin, ${ }^{\mathrm{a}}$ \\ Md. Sohug Sarker, ${ }^{a *}$ Mohammad Salim ${ }^{a}$ and Md. Mahbubul Alam ${ }^{a}$
}

${ }^{\mathbf{a}}$ Department of Chemistry, Shahjalal University of Science and Technology, Sylhet 3114, Bangladesh, ${ }^{\mathbf{b}}$ Department of Chemistry, Faculty of Science, Universiti Putra Malaysia, 43400 UPM Serdang, Selangor, Malaysia, and ${ }^{\mathbf{c}}$ School of Chemical Sciences, Universiti Sains Malaysia, Penang 11800 USM, Malaysia. *Correspondence e-mail: arafath.usm@gmail.com, sohug44@gmail.com

The molecule of the title compound, $\mathrm{C}_{28} \mathrm{H}_{22} \mathrm{~N}_{4} \mathrm{O}_{9}$, exhibits crystallographically imposed twofold rotational symmetry, with a dihedral angle of 66.0 (2) ${ }^{\circ}$ between the planes of the two central benzene rings bounded to the central oxygen atom. The dihedral angle between the planes of the central benzene ring and the terminal phenol ring is 4.9 (2) ${ }^{\circ}$. Each half of the molecule exhibits an imine $E$ configuration. An intramolecular $\mathrm{O}-\mathrm{H} \cdots \mathrm{N}$ hydrogen bond is present. In the crystal, the molecules are linked into layers parallel to the $a b$ plane via $\mathrm{C}-$ $\mathrm{H}$.. O hydrogen bonds. The crystal studied was refined as a two-component pseudomerohedral twin.

\section{Chemical context}

Bisthiosemicarbazones are formed by connecting separated thiosemicarbazone moieties through a pair of oxybisphenyl rings. These tetradentate ligands trap metals inside to form square-planar complexes (Alsop et al., 2005; Blower et al., 2003; Jasinski et al., 2003). The length of the $\mathrm{C}-\mathrm{C}$ bond in the backbone affects the stability of the complexes. A higher number of $\mathrm{C}-\mathrm{C}$ bonds obtained via alkylation or arylation allows metal ions to better fit inside the ligand cavity (Blower et al., 2003). These tetradentate ligands and transition-metal complexes exhibit promising anticancer and antibacterial activities (Lobana et al., 2009). In view of this and our research interest in the synthesis of oxybis Schiff base compounds, we herein report the crystal structure, supramolecular features and conformational comparison of the title compound.<smiles>COc1cc(/C=N/c2ccc(Oc3ccc(/N=C/c4cc(OC)cc([N+](=O)[O-])c4O)cc3)cc2)c(O)c([N+](=O)[O-])c1</smiles>

\section{Structural commentary}

In the title compound (Fig. 1), the asymmetric unit comprises one half of the oxybisbenzenyl molecule where the oxygen atom (O1) lies on a twofold rotation axis. The complete molecule is generated through the symmetry operation 


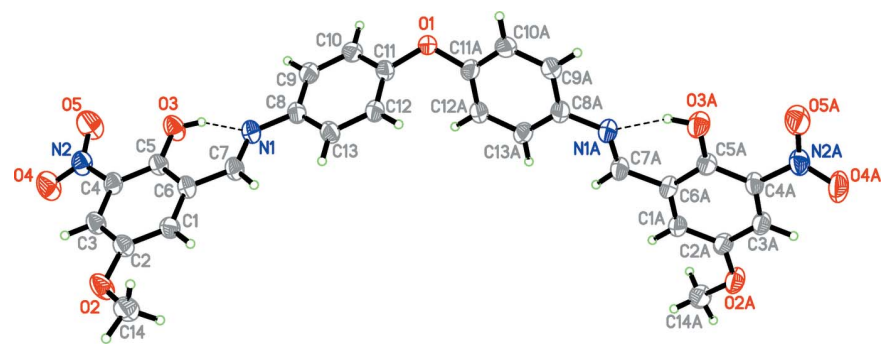

Figure 1

The molecular structure of the title compound with displacement ellipsoids drawn at the $50 \%$ probability level. Intramolecular hydrogen bonds are shown as dashed lines. Atoms with the label suffix A are generated by the symmetry operation $-x, y, \frac{1}{2}-z$.

$-x, y, \frac{1}{2}-z$. The planes of the benzene rings bonded to the central oxygen atom form a dihedral angle of $66.0(2)^{\circ}$. The dihedral angle between the benzene and 4-methoxy-2-nitrophenol rings in the same half of the molecules is $4.9(2)^{\circ}$, indicating an almost coplanar arrangement of the benzene and phenol rings. The $s p^{2}$-hybridized character of atoms $\mathrm{N} 1$ and $\mathrm{C} 7$ is confirmed by the $\mathrm{N} 1-\mathrm{C} 7[1.287(6) \AA]$ bond length and $\mathrm{C} 7-\mathrm{N} 1-\mathrm{C} 8\left[121.9(4)^{\circ}\right]$ and $\mathrm{N} 1-\mathrm{C} 7-\mathrm{C} 6\left[121.7(4)^{\circ}\right]$ bond angles (Arafath et al., 2018). Each half of the molecule exhibits an imine $E$ configuration with a $\mathrm{C} 6-\mathrm{C} 7-\mathrm{N} 1-\mathrm{C} 8$ torsion angle of $177.7(4)^{\circ}$. In the molecule, atom N1 of the imine moiety acts as a hydrogen-bond acceptor for the adjacent phenol group, forming an intramolecular $\mathrm{O}-\mathrm{H} \cdots \mathrm{N}$ hydrogen bond with an $S(6)$ ring motif (Fig. 1, Table 1).

\section{Supramolecular features}

In the crystal, atom $\mathrm{O} 5$ acts as a bifurcated-hydrogen-bond acceptor, linking molecules into layers parallel to the $a b$ plane (Fig. 2) through $\mathrm{C} 7-\mathrm{H} 7 A \cdots \mathrm{O} 5$ and $\mathrm{C} 13-\mathrm{H} 13 A \cdots \mathrm{O} 5$ hydrogen bonds (Table 1 ). No $\mathrm{C}-\mathrm{H} \cdots \pi$ or $\pi-\pi$ interactions are observed.

\section{Database survey}

In a search of the Cambridge Structure Database (CSD, version 5.40, last update August 2019; Groom et al., 2016), twelve structures containing the $\left(1 E, 1^{\prime} E\right)-N, N^{\prime}$-[oxybis $(4,1$ -
Table 1

Hydrogen-bond geometry $\left(\AA{ }^{\circ}\right)$.

\begin{tabular}{lllll}
\hline$D-\mathrm{H} \cdots A$ & $D-\mathrm{H}$ & $\mathrm{H} \cdots A$ & $D \cdots A$ & $D-\mathrm{H} \cdots A$ \\
\hline $\mathrm{O} 3-\mathrm{H} 1 O 3 \cdots \mathrm{N} 1$ & $0.85(9)$ & $1.81(10)$ & $2.591(6)$ & $153(7)$ \\
$\mathrm{C} 7-\mathrm{H} 7 A \cdots 5^{\mathrm{i}}$ & 0.95 & 2.54 & $3.470(7)$ & 167 \\
$\mathrm{C}^{2}-\mathrm{H} 13 A \cdots 5^{\mathrm{i}}$ & 0.95 & 2.48 & $3.404(7)$ & 165 \\
\hline
\end{tabular}

Symmetry code: (i) $x-\frac{1}{2}, y+\frac{1}{2}, z$.<smiles>[R]C=Nc1ccc(Oc2ccc(/N=C/[R])cc2)cc1</smiles>

Figure 3

Structural fragment for the CSD search.

phenylene)]bis(1-phenylmethanimine) moiety with different substituents were found. The reference moiety is illustrated in Fig. 3. Details regarding different substituents $\left(\mathbf{R}_{\mathbf{1}}\right)$ together with the dihedral and torsion angles for oxybisbenzenyl moiety in these structures are tabulated in Table 2. In analogy with the title molecule, the planes of the central benzene ring bonded to the central oxygen atom are always V-shaped with dihedral angle 1 in the range of 54.6-84. $8^{\circ}$. The dihedral angle between the planes of central and terminal benzene rings exists in two conformations, viz. non-coplanar [dihedral $2=$ $18.0-73.5^{\circ}$ ] and nearly coplanar [dihedral $2=4.8-9.9^{\circ}$ ]. In all of these structures, the imine $\mathrm{C}=\mathrm{N}$ double bond adopts an $E$ configuration with torsion angles corresponding to $\mathrm{C} 6-\mathrm{C} 7-$ $\mathrm{N} 1-\mathrm{C} 8$ in the range $172.9-180.0^{\circ}$.

\section{Synthesis and crystallization}

To a sample of 2-hydroxy-5-methoxy-3-nitrobenzaldehyde $(0.98 \mathrm{~g}, 5.00 \mathrm{mmol})$ dissolved in $25.0 \mathrm{~mL}$ of methanol, $0.20 \mathrm{~mL}$ of glacial acetic acid were added, and the mixture was refluxed for $30 \mathrm{~min}$. A solution of 4,4'-oxydianiline $(0.50 \mathrm{~g}, 2.50 \mathrm{mmol})$ in $20.0 \mathrm{~mL}$ of methanol was added dropwise under stirring to the aldehyde solution. The resulting deep-red solution was refluxed for $4 \mathrm{~h}$ with stirring. The reaction scheme is shown in Fig. 4. The deep-red precipitate that formed was filtered off and washed with $5.0 \mathrm{~mL}$ of methanol and $5.0 \mathrm{~mL}$ of $n$-hexane.

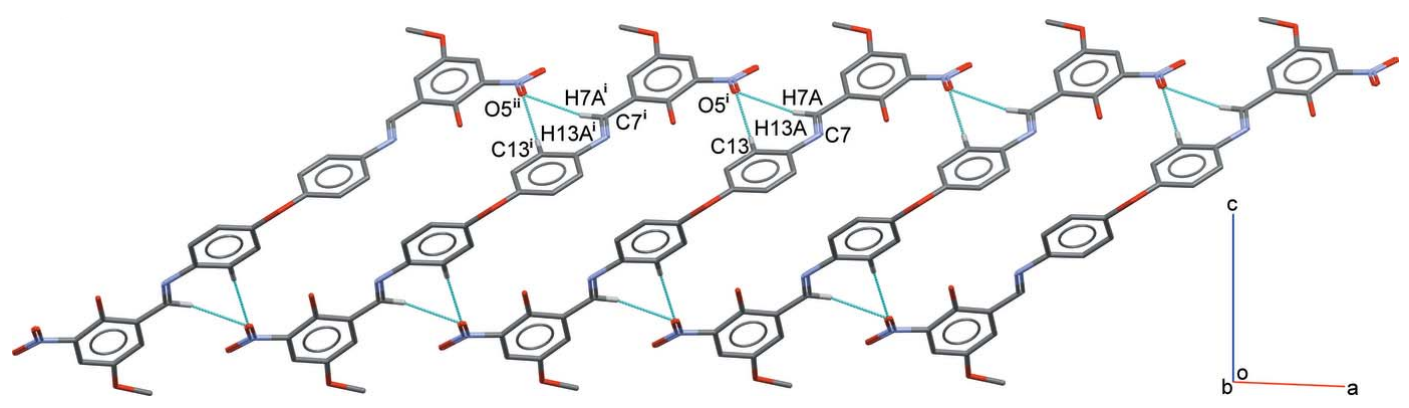

Figure 2

Partial packing diagram for the title compound, showing intermolecular hydrogen bonds (cyan dotted lines). Hydrogen atoms not involved in hydrogen bonding are omitted for clarity. Symmetry codes: (i) $-\frac{1}{2}+x, \frac{1}{2}+y, z$; (ii) $-1+x, 1+y, z$. 
Table 2

Selected dihedral and torsion angles $\left({ }^{\circ}\right)$.

Dihedral 1 is the dihedral angle between the planes of the central benzene rings. Dihedral 2 is the dihedral angle between the planes of the central and terminal benzene rings.

\begin{tabular}{|c|c|c|c|c|}
\hline Compound & $R_{1}$ & Dihedral 1 & Dihedral 2 & $\mathrm{C} 6-\mathrm{C} 7-\mathrm{N} 1-\mathrm{C} 8$ \\
\hline (I) & 4-methoxy-2-nitrophenol & $66.0(2)$ & $4.9(2), 4.9(2)$ & $-177.7(4),-177.7(4)$ \\
\hline DICKUW (Chu \& Huang, 2007) & 2,4-di-tert-butylphenol & 73.8 & $4.8,35.5$ & $178.2,177.2$ \\
\hline DICLAD (Chu \& Huang, 2007) & 2-(tert-butyl)-4-methylphenol & 73.8 & $47.9,46.3$ & $175.2,-179.9$ \\
\hline GIFCEG (Arafath et al., 2018) & 2-methylphenol & 59.5 & $36.0,31.5$ & $178.3,179.0$ \\
\hline HUDJEW (Lee \& Lee, 2009) & 4-nitrophenyl & 75.7 & $53.0,18.0$ & $-174.0,179.2$ \\
\hline NATWEM (Khalaji et al., 2012) & 2,3,4-trimethoxyphenyl & 84.8 & $57.6,73.1$ & $-179.2,-175.7$ \\
\hline PEHGOA (Kadu et al., 2013) & phenyl & 59.8 & $8.8,6.0$ & $-179.9,179.8$ \\
\hline PEHHAN (Kadu et al., 2013) & 4-methoxyphenyl & 60.1 & $5.3,5.3$ & $-179.3,-179.3$ \\
\hline RIZFEM (Xu et al., 2008) & 2-methoxyphenol & 69.2 & $24.3,24.3$ & $-180.0,-180.0$ \\
\hline TOWSOP (Kaabi et al., 2015) & 3-(diethylamino)phenol & 65.7 & $41.4,30.6$ & $-173.1,-176.5$ \\
\hline UNUFEP (Shahverdizadeh \& Tiekink, 2011) & phenol & 54.6 & $51.6,51.6$ & $173.5,173.4$ \\
\hline WEFLUQ (Krishna et al., 2012) & naphthalen-2-ol & $75.1 / 70.1$ & $7.7,9.9 / 6.1,19.4$ & $176.5,177.6 /-179.3,-172.9$ \\
\hline WIGPOT (Haffar et al., 2013) & naphthalen-2-ol & $74.6 / 69.9$ & 7.7. $9.9 / 19.6,5.8$ & $177.2,176.3 /-172.9,-178.6$ \\
\hline
\end{tabular}

Note: there is more than one data set for compounds WEFLUQ and WIGPOT because there is more than one independent molecule in their asymmetric units.

The recovered product was dissolved in chloroform for recrystallization. Purple single crystals suitable for X-ray diffraction were obtained by slow evaporation of the solvent, m.p. $547-548 \mathrm{~K}$, yield 96\%. Analysis calculated for $\mathrm{C}_{28} \mathrm{H}_{22} \mathrm{~N}_{4} \mathrm{O}_{9}$ (f.w. $558.50 \mathrm{~g} \mathrm{~mol}^{-1}$ ) C, 60.16; H, 3.93; N, 10; found: C, 59.04; $\mathrm{H}, 3.85 ; \mathrm{N}, 9.90 \% .{ }^{1} \mathrm{H} \mathrm{NMR}(500 \mathrm{MHz}$, DMSO- $d_{6}, \mathrm{Me}_{4} \mathrm{Si}$ ppm): $\delta 10.23(s, \mathrm{OH}), \delta 9.12(s, \mathrm{HC}=\mathrm{N}), \delta$ 7.69-7.21 (multiplet, aromatic), $\delta 3.83\left(s, \mathrm{Ph}-\mathrm{OCH}_{3}\right) .{ }^{13} \mathrm{C}$ NMR (DMSO- $\left.d_{6}, \mathrm{Me}_{4} \mathrm{Si} \mathrm{ppm}\right): \delta 161.69(\mathrm{C}=\mathrm{N}), \delta 156.21-$ 114.96 (C-aromatic), $\delta 56.25\left(\mathrm{OCH}_{3}\right)$. IR ( $\mathrm{KBr}$ pellets $v_{\max } /$ $\left.\mathrm{cm}^{-1}\right): 3441 v(\mathrm{OH}), 3109 v\left(\mathrm{C}-\mathrm{H}, s^{2}\right), 2956 v\left(\mathrm{CH}_{3}\right), 1598$ $v(\mathrm{C}=\mathrm{N}), 1529 v(\mathrm{C}=\mathrm{C}$, aromatic $), 1497 v\left(\mathrm{NO}_{2}\right.$, asym. $), 1326$ $v\left(\mathrm{NO}_{2}\right.$, sym. $), 1257 v(\mathrm{C}-\mathrm{O}$, phenolic $), 1194 v(\mathrm{C}-\mathrm{O}, \mathrm{Ph}-$ $\left.\mathrm{OCH}_{3}\right), 1056 v(\mathrm{C}-\mathrm{N}), 979 v(\mathrm{CH}$, bend. aromatic).

\section{Refinement}

Crystal data, data collection and structure refinement details are summarized in Table 3. The phenolic hydrogen atom was located in a difference-Fourier map and refined freely. All other $\mathrm{H}$ atoms attached to $\mathrm{C}$ were positioned geometrically and refined using a riding model with $\mathrm{C}-\mathrm{H}=0.95-0.98 \AA$ and $U_{\text {iso }}(\mathrm{H})=1.2 U_{\text {eq }}(\mathrm{C})$ or $1.5 U_{\text {eq }}(\mathrm{C})$ for methyl $\mathrm{H}$ atoms. A rotating model was used for the methyl group. The crystal investigated was refined as a two-component pseudomerohedral twin resulting from a $180^{\circ}$ rotation about the [001] reciprocal lattice direction, with a twin ratio of 0.977 (3):0.023 (3).

\section{Funding information}

The authors wish to thank Universiti Sains Malaysia and The World Academy of Sciences for a USM-TWAS fellowship to

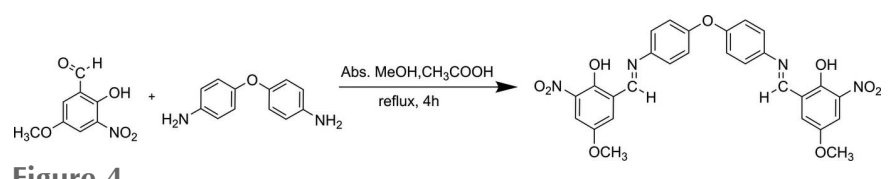

Figure 4

Reaction scheme for the synthesis of the title compound.
Md. Azharul Arafath. The research was supported financially by the RU grant 1001/PKIMIA/811269 from Universiti Sains Malaysia.

\section{References}

Alsop, L., Cowley, A. R., Dilworth, J. R., Donnelly, P. S., Peach, J. M. \& Rider, J. T. (2005). Inorg. Chim. Acta, 358, 2770-2780.

Arafath, M. A., Kwong, H. C., Adam, F. \& Razali, M. R. (2018). Acta Cryst. E74, 687-690.

Table 3

Experimental details.

\begin{tabular}{|c|c|}
\hline Crystal data & \\
\hline Chemical formula & $\mathrm{C}_{28} \mathrm{H}_{22} \mathrm{~N}_{4} \mathrm{O}_{9}$ \\
\hline$M_{\mathrm{r}}$ & 558.49 \\
\hline Crystal system, space group & Monoclinic, $C 2 / c$ \\
\hline Temperature $(\mathrm{K})$ & 100 \\
\hline$a, b, c(\AA)$ & $15.954(4), 5.4599(12), 28.397(6)$ \\
\hline$\beta\left({ }^{\circ}\right)$ & $92.299(5)$ \\
\hline$V\left(\AA^{3}\right)$ & 2471.7 (10) \\
\hline$Z$ & 4 \\
\hline Radiation type & Мо $K \alpha$ \\
\hline$\mu\left(\mathrm{mm}^{-1}\right)$ & 0.11 \\
\hline Crystal size $(\mathrm{mm})$ & $0.38 \times 0.24 \times 0.14$ \\
\hline
\end{tabular}

Data collection

Diffractometer

Absorption correction

$T_{\min }, T_{\max }$

No. of measured, independent and observed $[I>2 \sigma(I)]$ reflections

$R_{\text {int }}$
$(\sin \theta / \lambda)_{\max }\left(\AA^{-1}\right)$

Bruker APEX DUO CCD area
detector
Multi-scan ( $S A D A B S ;$ Bruker,
2012)
$0.879,0.956$
$35811,2830,2591$
0.038
0.650

$0.100,0.353,1.15$
2830
192
H atoms treated by a mixture of
$\quad$ independent and constrained
refinement
$0.31,-0.31$

Refinement

$R\left[F^{2}>2 \sigma\left(F^{2}\right)\right], w R\left(F^{2}\right), S$

No. of reflections

No. of parameters

$\mathrm{H}$-atom treatment

$\Delta \rho_{\max }, \Delta \rho_{\min }\left(\mathrm{e} \AA^{-3}\right)$ $0.31,-0.31$

Computer programs: APEX2 and SAINT (Bruker, 2012), SHELXS97 (Sheldrick, 2008), SHELXL2013 (Sheldrick, 2015), Mercury (Macrae et al., 2006) and PLATON (Spek, 2009). 
Blower, P. J., Castle, T. C., Cowley, A. R., Dilworth, J. R., Donnelly, P. S., Labisbal, E., Sowrey, F. E., Teat, S. J. \& Went, M. J. (2003). Dalton Trans. pp. 4416-4425.

Bruker (2012). APEX2, SAINT and SADABS. Bruker AXS Inc., Madison, Wisconsin, USA.

Chu, Z. \& Huang, W. (2007). J. Mol. Struct. 837, 15-22.

Groom, C. R., Bruno, I. J., Lightfoot, M. P. \& Ward, S. C. (2016). Acta Cryst. B72, 171-179.

Haffar, D., Daoud, D., Douadi, T., Bouzidi, L. \& Chafaa, S. (2013). Acta Cryst. E69, o581-0582.

Jasinski, J. P., Bianchani, J. R., Cueva, J., El-Saied, F. A., El-Asmy, A. A. \& West, D. X. (2003). Z. Anorg. Allg. Chem. 629, 202206.

Kaabi, I., Sibous, L., Douadi, T. \& Chafaa, S. (2015). J. Mol. Struct. 1084, 216-222.

Kadu, R., Singh, V. K., Verma, S. K., Raghavaiah, P. \& Shaikh, M. M. (2013). J. Mol. Struct. 1033, 298-311.
Khalaji, A. D., Fejfarova, K. \& Dusek, M. (2012). J. Chem. Crystallogr. 42, 263-266.

Krishna, V., Basavoju, S. \& Ramachandraiah, A. (2012). Mol. Cryst. Liq. Cryst. 562, 265-290.

Lee, H. K. \& Lee, S. W. (2009). Acta Cryst. E65, o2263.

Lobana, T. S., Sharma, R., Bawa, G. \& Khanna, S. (2009). Coord. Chem. Rev. 253, 977-1055.

Macrae, C. F., Edgington, P. R., McCabe, P., Pidcock, E., Shields, G. P., Taylor, R., Towler, M. \& van de Streek, J. (2006). J. Appl. Cryst. 39, 453-457.

Shahverdizadeh, G. H. \& Tiekink, E. R. T. (2011). Acta Cryst. E67, o798.

Sheldrick, G. M. (2008). Acta Cryst. A64, 112-122.

Sheldrick, G. M. (2015). Acta Cryst. C71, 3-8.

Spek, A. L. (2009). Acta Cryst. D65, 148-155.

Xu, H.-W., Li, J.-X. \& Li, Y.-H. (2008). Acta Cryst. E64, o1145. 


\section{supporting information}

Acta Cryst. (2020). E76, 91-94 [https://doi.org/10.1107/S2056989019016852]

Bis\{4-[(2-hydroxy-5-methoxy-3-nitrobenzylidene)amino] phenyl\} ether

Md. Azharul Arafath, Huey Chong Kwong, Farook Adam, Md. Mohiuddin, Md. Sohug Sarker, Mohammad Salim and Md. Mahbubul Alam

Computing details

Data collection: APEX2 (Bruker, 2012); cell refinement: SAINT (Bruker, 2012); data reduction: SAINT (Bruker, 2012); program(s) used to solve structure: SHELXS97 (Sheldrick, 2008); program(s) used to refine structure: SHELXL2013 (Sheldrick, 2015); molecular graphics: SHELXL2013 (Sheldrick, 2015) and Mercury (Macrae et al., 2006); software used to prepare material for publication: SHELXL2013 (Sheldrick, 2015) and PLATON (Spek, 2009).

2-[N-(4-\{4-[(2-Hydroxy-5-methoxy-3-nitrobenzylidene)amino] phenoxy\}phenyl)carboximidoyl]-4-methoxy-6-

nitrophenol

Crystal data

$\mathrm{C}_{28} \mathrm{H}_{22} \mathrm{~N}_{4} \mathrm{O}_{9}$

$M_{r}=558.49$

Monoclinic, $C 2 / c$

$a=15.954(4) \AA$

$b=5.4599(12) \AA$

$c=28.397(6) \AA$

$\beta=92.299(5)^{\circ}$

$V=2471.7(10) \AA^{3}$

$Z=4$

\section{Data collection}

Bruker APEX DUO CCD area detector diffractometer

Radiation source: fine-focus sealed tube Graphite monochromator $\varphi$ and $\omega$ scans

Absorption correction: multi-scan

(SADABS; Bruker, 2012)

$T_{\min }=0.879, T_{\max }=0.956$

\section{Refinement}

Refinement on $F^{2}$

Least-squares matrix: full

$R\left[F^{2}>2 \sigma\left(F^{2}\right)\right]=0.100$

$w R\left(F^{2}\right)=0.353$

$S=1.15$

2830 reflections

192 parameters

0 restraints
$F(000)=1160$

$D_{\mathrm{x}}=1.501 \mathrm{Mg} \mathrm{m}^{-3}$

Mo $K \alpha$ radiation, $\lambda=0.71073 \AA$

Cell parameters from 9905 reflections

$\theta=3-31^{\circ}$

$\mu=0.11 \mathrm{~mm}^{-1}$

$T=100 \mathrm{~K}$

Block, purple

$0.38 \times 0.24 \times 0.14 \mathrm{~mm}$

35811 measured reflections

2830 independent reflections

2591 reflections with $I>2 \sigma(I)$

$R_{\text {int }}=0.038$

$\theta_{\max }=27.5^{\circ}, \theta_{\min }=0.7^{\circ}$

$h=-20 \rightarrow 20$

$k=-7 \rightarrow 7$

$l=-36 \rightarrow 36$

Hydrogen site location: mixed

$\mathrm{H}$ atoms treated by a mixture of independent and constrained refinement

$w=1 /\left[\sigma^{2}\left(F_{\mathrm{o}}^{2}\right)+(0.1539 P)^{2}+17.7934 P\right]$ where $P=\left(F_{\mathrm{o}}^{2}+2 F_{\mathrm{c}}^{2}\right) / 3$

$(\Delta / \sigma)_{\max }<0.001$

$\Delta \rho_{\max }=0.31$ e $\AA^{-3}$

$\Delta \rho_{\min }=-0.31 \mathrm{e} \AA^{-3}$ 


\section{Special details}

Experimental. The following wavelength and cell were deduced by SADABS from the direction cosines etc. They are given here for emergency use only: CELL 0.710955 .4638 .44328 .41892 .10689 .981108 .897

Geometry. All esds (except the esd in the dihedral angle between two 1.s. planes) are estimated using the full covariance matrix. The cell esds are taken into account individually in the estimation of esds in distances, angles and torsion angles; correlations between esds in cell parameters are only used when they are defined by crystal symmetry. An approximate (isotropic) treatment of cell esds is used for estimating esds involving l.s. planes.

Refinement. Refined as a 2-component twin.

Fractional atomic coordinates and isotropic or equivalent isotropic displacement parameters $\left(\hat{A}^{2}\right)$

\begin{tabular}{|c|c|c|c|c|}
\hline & $x$ & $y$ & $z$ & $U_{\text {iso }} * / U_{\text {eq }}$ \\
\hline O1 & 0.000000 & $-0.2692(9)$ & 0.250000 & $0.0444(13)$ \\
\hline $\mathrm{O} 2$ & $0.4044(2)$ & $0.9524(8)$ & $0.47761(16)$ & $0.0525(11)$ \\
\hline $\mathrm{O} 3$ & $0.4346(3)$ & $0.1480(8)$ & $0.36154(14)$ & $0.0475(10)$ \\
\hline $\mathrm{O} 4$ & $0.6359(2)$ & $0.4545(9)$ & $0.43220(19)$ & $0.0664(14)$ \\
\hline $\mathrm{O} 5$ & $0.5853(3)$ & $0.1292(9)$ & $0.4007(2)$ & $0.0710(15)$ \\
\hline N1 & $0.2747(2)$ & $0.1732(8)$ & $0.34224(14)$ & $0.0371(9)$ \\
\hline N2 & $0.5771(3)$ & $0.3336(9)$ & $0.41607(16)$ & $0.0417(10)$ \\
\hline $\mathrm{C} 1$ & 0.3380 & $0.6629(9)$ & $0.42249(18)$ & $0.0364(10)$ \\
\hline $\mathrm{H} 1 \mathrm{~A}$ & 0.284746 & 0.739136 & 0.424432 & $0.044 *$ \\
\hline $\mathrm{C} 2$ & $0.4061(3)$ & $0.7544(9)$ & $0.44839(17)$ & $0.0355(10)$ \\
\hline C3 & $0.4833(3)$ & $0.6438(9)$ & $0.44506(17)$ & $0.0365(10)$ \\
\hline H3A & 0.530272 & 0.707394 & 0.462653 & $0.044 *$ \\
\hline $\mathrm{C} 4$ & $0.4934(3)$ & $0.4407(9)$ & $0.41635(16)$ & $0.0337(10)$ \\
\hline $\mathrm{C} 5$ & 0.4255 & $0.3424(9)$ & $0.38929(16)$ & $0.0332(10)$ \\
\hline C6 & $0.3471(3)$ & $0.4586(9)$ & $0.39343(16)$ & $0.0337(10)$ \\
\hline $\mathrm{C} 7$ & $0.2723(3)$ & $0.3645(9)$ & $0.36861(17)$ & $0.0366(10)$ \\
\hline H7A & 0.220289 & 0.446315 & 0.371966 & $0.044 *$ \\
\hline $\mathrm{C} 8$ & 0.2016 & $0.0754(9)$ & $0.31932(16)$ & $0.0335(10)$ \\
\hline C9 & $0.2115(3)$ & $-0.1371(9)$ & $0.29336(17)$ & $0.0366(10)$ \\
\hline H9A & 0.265646 & -0.208522 & 0.291709 & $0.044 *$ \\
\hline C10 & $0.1439(3)$ & $-0.2462(9)$ & $0.26992(16)$ & $0.0369(10)$ \\
\hline H10A & 0.151248 & -0.392646 & 0.252498 & $0.044 *$ \\
\hline C11 & $0.0657(3)$ & $-0.1405(9)$ & $0.27200(16)$ & $0.0349(10)$ \\
\hline C12 & 0.0535 & $0.0722(9)$ & $0.29753(18)$ & $0.0395(11)$ \\
\hline H12A & -0.000735 & 0.142841 & 0.298889 & $0.047^{*}$ \\
\hline C13 & $0.1217(3)$ & $0.1799(9)$ & $0.32098(17)$ & $0.0386(11)$ \\
\hline H13A & 0.114209 & 0.326303 & 0.338386 & $0.046^{*}$ \\
\hline C14 & $0.3252(3)$ & $1.0498(11)$ & $0.4876(2)$ & 0.0477 (13) \\
\hline H14A & 0.332369 & 1.185096 & 0.510095 & $0.072 *$ \\
\hline H14B & 0.290647 & 0.921682 & 0.501263 & $0.072 *$ \\
\hline $\mathrm{H} 14 \mathrm{C}$ & 0.297545 & 1.110264 & 0.458451 & $0.072^{*}$ \\
\hline $\mathrm{H} 1 \mathrm{O} 3$ & $0.386(6)$ & $0.117(15)$ & $0.350(3)$ & $0.08(3)^{*}$ \\
\hline
\end{tabular}


Atomic displacement parameters $\left(\AA^{2}\right)$

\begin{tabular}{lllllll}
\hline & $U^{11}$ & $U^{22}$ & $U^{33}$ & $U^{12}$ & $U^{13}$ & $U^{23}$ \\
\hline O1 & $0.047(3)$ & $0.029(2)$ & $0.055(3)$ & 0.000 & $-0.023(2)$ & 0.000 \\
O2 & $0.0300(17)$ & $0.054(2)$ & $0.073(3)$ & $0.0037(16)$ & $-0.0050(16)$ & $-0.031(2)$ \\
O3 & $0.0378(19)$ & $0.054(2)$ & $0.050(2)$ & $0.0037(16)$ & $-0.0020(15)$ & $-0.0231(18)$ \\
O4 & $0.0290(18)$ & $0.074(3)$ & $0.095(3)$ & $0.0049(19)$ & $-0.013(2)$ & $-0.024(3)$ \\
O5 & $0.044(2)$ & $0.069(3)$ & $0.099(4)$ & $0.018(2)$ & $-0.008(2)$ & $-0.039(3)$ \\
N1 & $0.0291(18)$ & $0.043(2)$ & $0.039(2)$ & $-0.0034(16)$ & $-0.0033(15)$ & $-0.0025(17)$ \\
N2 & $0.0296(19)$ & $0.052(2)$ & $0.044(2)$ & $0.0046(18)$ & $0.0000(16)$ & $-0.0073(19)$ \\
C1 & $0.027(2)$ & $0.035(2)$ & $0.047(2)$ & $0.0005(17)$ & $-0.0033(18)$ & $-0.003(2)$ \\
C2 & $0.030(2)$ & $0.037(2)$ & $0.039(2)$ & $-0.0011(18)$ & $0.0016(17)$ & $-0.0075(19)$ \\
C3 & $0.027(2)$ & $0.041(2)$ & $0.042(2)$ & $-0.0031(18)$ & $-0.0018(17)$ & $-0.006(2)$ \\
C4 & $0.0241(19)$ & $0.041(2)$ & $0.036(2)$ & $0.0020(17)$ & $-0.0006(16)$ & $-0.0026(18)$ \\
C5 & $0.030(2)$ & $0.037(2)$ & $0.033(2)$ & $-0.0002(18)$ & $0.0015(16)$ & $-0.0051(18)$ \\
C6 & $0.028(2)$ & $0.040(2)$ & $0.033(2)$ & $-0.0040(18)$ & $-0.0020(16)$ & $-0.0031(18)$ \\
C7 & $0.027(2)$ & $0.043(3)$ & $0.040(2)$ & $-0.0021(18)$ & $-0.0027(17)$ & $-0.003(2)$ \\
C8 & $0.031(2)$ & $0.037(2)$ & $0.032(2)$ & $-0.0019(18)$ & $-0.0028(16)$ & $0.0004(18)$ \\
C9 & $0.034(2)$ & $0.036(2)$ & $0.039(2)$ & $0.0035(18)$ & $-0.0025(18)$ & $-0.0007(19)$ \\
C10 & $0.042(2)$ & $0.033(2)$ & $0.035(2)$ & $0.0023(19)$ & $-0.0030(18)$ & $-0.0017(18)$ \\
C11 & $0.039(2)$ & $0.034(2)$ & $0.032(2)$ & $-0.0052(18)$ & $-0.0086(17)$ & $0.0028(18)$ \\
C12 & $0.034(2)$ & $0.037(2)$ & $0.046(3)$ & $0.0053(19)$ & $-0.0107(19)$ & $-0.004(2)$ \\
C13 & $0.037(2)$ & $0.038(2)$ & $0.041(2)$ & $0.0022(19)$ & $-0.0072(18)$ & $-0.011(2)$ \\
C14 & $0.037(2)$ & $0.046(3)$ & $0.060(3)$ & $0.007(2)$ & $0.003(2)$ & $-0.017(3)$ \\
& & & & & & \\
& & & & & &
\end{tabular}

Geometric parameters $\left(\AA,{ }^{\circ}\right)$

\begin{tabular}{llll}
\hline $\mathrm{O} 1-\mathrm{C} 11^{\mathrm{i}}$ & $1.389(5)$ & $\mathrm{C} 4-\mathrm{C} 5$ & $1.409(6)$ \\
$\mathrm{O} 1-\mathrm{C} 11$ & $1.389(5)$ & $\mathrm{C} 5-\mathrm{C} 6$ & $1.412(6)$ \\
$\mathrm{O} 2-\mathrm{C} 2$ & $1.364(6)$ & $\mathrm{C} 6-\mathrm{C} 7$ & $1.456(6)$ \\
$\mathrm{O} 2-\mathrm{C} 14$ & $1.410(6)$ & $\mathrm{C} 7-\mathrm{H} 7 \mathrm{~A}$ & 0.9500 \\
$\mathrm{O} 3-\mathrm{C} 5$ & $1.333(6)$ & $\mathrm{C} 8-\mathrm{C} 9$ & $1.387(7)$ \\
$\mathrm{O} 3-\mathrm{H} 1 \mathrm{O} 3$ & $0.85(9)$ & $\mathrm{C} 8-\mathrm{C} 13$ & $1.398(6)$ \\
$\mathrm{O} 4-\mathrm{N} 2$ & $1.221(6)$ & $\mathrm{C} 9-\mathrm{C} 10$ & $1.380(7)$ \\
$\mathrm{O} 5-\mathrm{N} 2$ & $1.207(6)$ & $\mathrm{C} 9-\mathrm{H} 9 \mathrm{~A}$ & 0.9500 \\
$\mathrm{~N} 1-\mathrm{C} 7$ & $1.287(6)$ & $\mathrm{C} 10-\mathrm{C} 11$ & $1.379(7)$ \\
$\mathrm{N} 1-\mathrm{C} 8$ & $1.418(6)$ & $\mathrm{C} 10-\mathrm{H} 10 \mathrm{~A}$ & 0.9500 \\
$\mathrm{~N} 2-\mathrm{C} 4$ & $1.458(6)$ & $\mathrm{C} 11-\mathrm{C} 12$ & $1.387(7)$ \\
$\mathrm{C} 1-\mathrm{C} 2$ & $1.381(6)$ & $\mathrm{C} 12-\mathrm{C} 13$ & $1.384(6)$ \\
$\mathrm{C} 1-\mathrm{C} 6$ & $1.398(7)$ & $\mathrm{C} 12-\mathrm{H} 12 \mathrm{~A}$ & 0.9500 \\
$\mathrm{C} 1-\mathrm{H} 1 \mathrm{~A}$ & 0.9500 & $\mathrm{C} 13-\mathrm{H} 13 \mathrm{~A}$ & 0.9500 \\
$\mathrm{C} 2-\mathrm{C} 3$ & $1.377(6)$ & $\mathrm{C} 14-\mathrm{H} 14 \mathrm{~A}$ & 0.9800 \\
$\mathrm{C} 3-\mathrm{C} 4$ & $1.390(7)$ & $\mathrm{C} 14-\mathrm{H} 14 \mathrm{~B}$ & 0.9800 \\
$\mathrm{C} 3-\mathrm{H} 3 \mathrm{~A}$ & 0.9500 & $\mathrm{C} 14-\mathrm{H} 14 \mathrm{C}$ & 0.9800 \\
& & & 119.2 \\
$\mathrm{C} 11^{\mathrm{i}}-\mathrm{O} 1-\mathrm{C} 11$ & $119.2(5)$ & $\mathrm{N} 1-\mathrm{C} 7-\mathrm{H} 7 \mathrm{~A}$ & 119.2 \\
$\mathrm{C} 2-\mathrm{O} 2-\mathrm{C} 14$ & $117.5(4)$ & $\mathrm{C} 6-\mathrm{C} 7-\mathrm{H} 7 \mathrm{~A}$ & $118.9(4)$ \\
$\mathrm{C} 5-\mathrm{O} 3-\mathrm{H} 1 \mathrm{O} 3$ & $106(6)$ & $\mathrm{C} 9-\mathrm{C} 8-\mathrm{C} 13$ &
\end{tabular}




\begin{tabular}{|c|c|c|c|}
\hline $\mathrm{C} 7-\mathrm{N} 1-\mathrm{C} 8$ & $121.9(4)$ & $\mathrm{C} 9-\mathrm{C} 8-\mathrm{N} 1$ & $116.7(4)$ \\
\hline $\mathrm{O} 5-\mathrm{N} 2-\mathrm{O} 4$ & $122.8(5)$ & $\mathrm{C} 13-\mathrm{C} 8-\mathrm{N} 1$ & $124.4(4)$ \\
\hline $\mathrm{O} 5-\mathrm{N} 2-\mathrm{C} 4$ & $119.0(4)$ & $\mathrm{C} 10-\mathrm{C} 9-\mathrm{C} 8$ & $120.9(4)$ \\
\hline $\mathrm{O} 4-\mathrm{N} 2-\mathrm{C} 4$ & $118.1(4)$ & $\mathrm{C} 10-\mathrm{C} 9-\mathrm{H} 9 \mathrm{~A}$ & 119.6 \\
\hline $\mathrm{C} 2-\mathrm{C} 1-\mathrm{C} 6$ & $120.3(4)$ & $\mathrm{C} 8-\mathrm{C} 9-\mathrm{H} 9 \mathrm{~A}$ & 119.6 \\
\hline $\mathrm{C} 2-\mathrm{C} 1-\mathrm{H} 1 \mathrm{~A}$ & 119.8 & $\mathrm{C} 11-\mathrm{C} 10-\mathrm{C} 9$ & $119.4(4)$ \\
\hline $\mathrm{C} 6-\mathrm{C} 1-\mathrm{H} 1 \mathrm{~A}$ & 119.8 & $\mathrm{C} 11-\mathrm{C} 10-\mathrm{H} 10 \mathrm{~A}$ & 120.3 \\
\hline $\mathrm{O} 2-\mathrm{C} 2-\mathrm{C} 3$ & $115.4(4)$ & $\mathrm{C} 9-\mathrm{C} 10-\mathrm{H} 10 \mathrm{~A}$ & 120.3 \\
\hline $\mathrm{O} 2-\mathrm{C} 2-\mathrm{C} 1$ & $125.2(4)$ & $\mathrm{C} 10-\mathrm{C} 11-\mathrm{C} 12$ & $121.2(4)$ \\
\hline $\mathrm{C} 3-\mathrm{C} 2-\mathrm{C} 1$ & $119.4(4)$ & $\mathrm{C} 10-\mathrm{C} 11-\mathrm{O} 1$ & $115.9(4)$ \\
\hline $\mathrm{C} 2-\mathrm{C} 3-\mathrm{C} 4$ & $121.0(4)$ & $\mathrm{C} 12-\mathrm{C} 11-\mathrm{O} 1$ & $122.7(4)$ \\
\hline $\mathrm{C} 2-\mathrm{C} 3-\mathrm{H} 3 \mathrm{~A}$ & 119.5 & $\mathrm{C} 13-\mathrm{C} 12-\mathrm{C} 11$ & $118.9(4)$ \\
\hline $\mathrm{C} 4-\mathrm{C} 3-\mathrm{H} 3 \mathrm{~A}$ & 119.5 & $\mathrm{C} 13-\mathrm{C} 12-\mathrm{H} 12 \mathrm{~A}$ & 120.5 \\
\hline $\mathrm{C} 3-\mathrm{C} 4-\mathrm{C} 5$ & $121.3(4)$ & $\mathrm{C} 11-\mathrm{C} 12-\mathrm{H} 12 \mathrm{~A}$ & 120.5 \\
\hline $\mathrm{C} 3-\mathrm{C} 4-\mathrm{N} 2$ & $116.8(4)$ & $\mathrm{C} 12-\mathrm{C} 13-\mathrm{C} 8$ & $120.7(4)$ \\
\hline $\mathrm{C} 5-\mathrm{C} 4-\mathrm{N} 2$ & $121.9(4)$ & $\mathrm{C} 12-\mathrm{C} 13-\mathrm{H} 13 \mathrm{~A}$ & 119.7 \\
\hline $\mathrm{O} 3-\mathrm{C} 5-\mathrm{C} 4$ & $121.8(4)$ & $\mathrm{C} 8-\mathrm{C} 13-\mathrm{H} 13 \mathrm{~A}$ & 119.7 \\
\hline $\mathrm{O} 3-\mathrm{C} 5-\mathrm{C} 6$ & $121.6(4)$ & $\mathrm{O} 2-\mathrm{C} 14-\mathrm{H} 14 \mathrm{~A}$ & 109.5 \\
\hline $\mathrm{C} 4-\mathrm{C} 5-\mathrm{C} 6$ & $116.6(4)$ & $\mathrm{O} 2-\mathrm{C} 14-\mathrm{H} 14 \mathrm{~B}$ & 109.5 \\
\hline $\mathrm{C} 1-\mathrm{C} 6-\mathrm{C} 5$ & $121.4(4)$ & $\mathrm{H} 14 \mathrm{~A}-\mathrm{C} 14-\mathrm{H} 14 \mathrm{~B}$ & 109.5 \\
\hline $\mathrm{C} 1-\mathrm{C} 6-\mathrm{C} 7$ & $117.7(4)$ & $\mathrm{O} 2-\mathrm{C} 14-\mathrm{H} 14 \mathrm{C}$ & 109.5 \\
\hline $\mathrm{C} 5-\mathrm{C} 6-\mathrm{C} 7$ & $120.9(4)$ & $\mathrm{H} 14 \mathrm{~A}-\mathrm{C} 14-\mathrm{H} 14 \mathrm{C}$ & 109.5 \\
\hline $\mathrm{N} 1-\mathrm{C} 7-\mathrm{C} 6$ & $121.7(4)$ & $\mathrm{H} 14 \mathrm{~B}-\mathrm{C} 14-\mathrm{H} 14 \mathrm{C}$ & 109.5 \\
\hline $\mathrm{C} 14-\mathrm{O} 2-\mathrm{C} 2-\mathrm{C} 3$ & $-170.9(5)$ & $\mathrm{O} 3-\mathrm{C} 5-\mathrm{C} 6-\mathrm{C} 7$ & $2.1(7)$ \\
\hline $\mathrm{C} 14-\mathrm{O} 2-\mathrm{C} 2-\mathrm{C} 1$ & $9.8(8)$ & $\mathrm{C} 4-\mathrm{C} 5-\mathrm{C} 6-\mathrm{C} 7$ & $-177.2(4)$ \\
\hline $\mathrm{C} 6-\mathrm{C} 1-\mathrm{C} 2-\mathrm{O} 2$ & $179.7(5)$ & $\mathrm{C} 8-\mathrm{N} 1-\mathrm{C} 7-\mathrm{C} 6$ & $177.7(4)$ \\
\hline $\mathrm{C} 6-\mathrm{C} 1-\mathrm{C} 2-\mathrm{C} 3$ & $0.5(8)$ & $\mathrm{C} 1-\mathrm{C} 6-\mathrm{C} 7-\mathrm{N} 1$ & $-178.0(5)$ \\
\hline $\mathrm{O} 2-\mathrm{C} 2-\mathrm{C} 3-\mathrm{C} 4$ & $-179.8(5)$ & $\mathrm{C} 5-\mathrm{C} 6-\mathrm{C} 7-\mathrm{N} 1$ & $0.1(7)$ \\
\hline $\mathrm{C} 1-\mathrm{C} 2-\mathrm{C} 3-\mathrm{C} 4$ & $-0.5(8)$ & $\mathrm{C} 7-\mathrm{N} 1-\mathrm{C} 8-\mathrm{C} 9$ & $-177.5(4)$ \\
\hline $\mathrm{C} 2-\mathrm{C} 3-\mathrm{C} 4-\mathrm{C} 5$ & $0.7(7)$ & $\mathrm{C} 7-\mathrm{N} 1-\mathrm{C} 8-\mathrm{C} 13$ & $3.2(8)$ \\
\hline $\mathrm{C} 2-\mathrm{C} 3-\mathrm{C} 4-\mathrm{N} 2$ & $-178.6(5)$ & $\mathrm{C} 13-\mathrm{C} 8-\mathrm{C} 9-\mathrm{C} 10$ & $-0.7(7)$ \\
\hline $\mathrm{O} 5-\mathrm{N} 2-\mathrm{C} 4-\mathrm{C} 3$ & $163.3(5)$ & $\mathrm{N} 1-\mathrm{C} 8-\mathrm{C} 9-\mathrm{C} 10$ & $179.9(4)$ \\
\hline $\mathrm{O} 4-\mathrm{N} 2-\mathrm{C} 4-\mathrm{C} 3$ & $-15.5(7)$ & $\mathrm{C} 8-\mathrm{C} 9-\mathrm{C} 10-\mathrm{C} 11$ & $0.7(7)$ \\
\hline $\mathrm{O} 5-\mathrm{N} 2-\mathrm{C} 4-\mathrm{C} 5$ & $-16.1(8)$ & $\mathrm{C} 9-\mathrm{C} 10-\mathrm{C} 11-\mathrm{C} 12$ & $-0.5(7)$ \\
\hline $\mathrm{O} 4-\mathrm{N} 2-\mathrm{C} 4-\mathrm{C} 5$ & $165.1(5)$ & $\mathrm{C} 9-\mathrm{C} 10-\mathrm{C} 11-\mathrm{O} 1$ & $-176.1(4)$ \\
\hline $\mathrm{C} 3-\mathrm{C} 4-\mathrm{C} 5-\mathrm{O} 3$ & $179.8(5)$ & $\mathrm{C} 11^{\mathrm{i}}-\mathrm{O} 1-\mathrm{C} 11-\mathrm{C} 10$ & $-145.5(5)$ \\
\hline $\mathrm{N} 2-\mathrm{C} 4-\mathrm{C} 5-\mathrm{O} 3$ & $-0.9(7)$ & $\mathrm{C} 11^{\mathrm{i}}-\mathrm{O} 1-\mathrm{C} 11-\mathrm{C} 12$ & $39.0(4)$ \\
\hline $\mathrm{C} 3-\mathrm{C} 4-\mathrm{C} 5-\mathrm{C} 6$ & $-0.8(7)$ & $\mathrm{C} 10-\mathrm{C} 11-\mathrm{C} 12-\mathrm{C} 13$ & $0.4(8)$ \\
\hline $\mathrm{N} 2-\mathrm{C} 4-\mathrm{C} 5-\mathrm{C} 6$ & $178.5(4)$ & $\mathrm{O} 1-\mathrm{C} 11-\mathrm{C} 12-\mathrm{C} 13$ & $175.7(4)$ \\
\hline $\mathrm{C} 2-\mathrm{C} 1-\mathrm{C} 6-\mathrm{C} 5$ & $-0.7(7)$ & $\mathrm{C} 11-\mathrm{C} 12-\mathrm{C} 13-\mathrm{C} 8$ & $-0.5(8)$ \\
\hline $\mathrm{C} 2-\mathrm{C} 1-\mathrm{C} 6-\mathrm{C} 7$ & $177.4(5)$ & $\mathrm{C} 9-\mathrm{C} 8-\mathrm{C} 13-\mathrm{C} 12$ & $0.6(8)$ \\
\hline $\mathrm{O} 3-\mathrm{C} 5-\mathrm{C} 6-\mathrm{C} 1$ & $-179.8(5)$ & $\mathrm{N} 1-\mathrm{C} 8-\mathrm{C} 13-\mathrm{C} 12$ & $180.0(5)$ \\
\hline $\mathrm{C} 4-\mathrm{C} 5-\mathrm{C} 6-\mathrm{C} 1$ & $0.8(7)$ & & \\
\hline
\end{tabular}

Symmetry code: (i) $-x, y,-z+1 / 2$. 
supporting information

Hydrogen-bond geometry $\left(\AA,{ }^{\circ}\right)$

\begin{tabular}{lllll}
\hline$D-\mathrm{H} \cdots A$ & $D-\mathrm{H}$ & $\mathrm{H} \cdots A$ & $D \cdots A$ & $D-\mathrm{H} \cdots A$ \\
\hline $\mathrm{O} 3-\mathrm{H} 1 O 3 \cdots \mathrm{N} 1$ & $0.85(9)$ & $1.81(10)$ & $2.591(6)$ & $153(7)$ \\
$\mathrm{C} 7-\mathrm{H} 7 A \cdots 5^{\mathrm{ii}}$ & 0.95 & 2.54 & $3.470(7)$ & 167 \\
$\mathrm{C} 13-\mathrm{H} 13 A \cdots \mathrm{O} 5^{\mathrm{ii}}$ & 0.95 & 2.48 & $3.404(7)$ & 165 \\
\hline
\end{tabular}

Symmetry code: (ii) $x-1 / 2, y+1 / 2, z$. 